\title{
APROXIMACIONES A LA SUBJETIVACIÓN SEXO-GENÉRICA EN LA PROFESIÓN DOCENTE. ESTUDIO COMPARATIVO DE TRES CURSOS DE FORMACION DOCENTE EN EDUCACION SEXUAL EN CORDOBA
}

\author{
Tomás losa (UNC-CONICET)*
}

\section{Resumen}

Este artículo presenta resultados de una investigación que abordó el campo de la formación continua de docentes en educación sexual posterior a la ley 26.150, Programa de Educación Sexual Integral (PESI). El objetivo primordial consiste en mapear el tipo de identidades socio-sexuales privilegiadas y aquellas deslegitimadas por los contenidos curriculares de tres cursos de formación docente en Educación Sexual dictados en la ciudad de Córdoba durante el período 2007-2008. La generación y el análisis de datos permiten en este estudio teorizar la práctica de la formación de docentes como el escenario de un proceso transformativo de identidades sociosexuales - vehiculizadas en los contenidos curriculares - en subjetividades sexogenérico-profesionales. Este proceso de subjetivación puede consolidar las formas consensuadas de legitimación profesional o cuestionarlas para reinventar la profesión y sus marcas sexuales y genéricas. El carácter del estudio es pues comparativo y los métodos utilizados para la generación de datos fueron la observación participante y el análisis de contenido del material impreso.

\section{Palabras clave}

Educación sexual - Formación docente - Identidad profesional - Subjetivación - Sexualidades.

\section{Abstract}

This article presents the partial results of a research study project that entailed the field of teacher's continuing training in sex education, posterior to act 26,150 'Program

\footnotetext{
Master en Sciences Humaines et Sociales, mention philosophie à finalité recherche. Université de Rennes 1 (Francia). Licenciado en Filosofía. Universidad Nacional de Córdoba. Becario Doctoral del CONICET (Tipo II)
} 
of Integral Sex Education' (PESI, for its acronym in Spanish). The main objective consists in mapping the types of socio-sexual identities that are privileged and those that are discredited in the curricula contents present in three courses of continuing training in Sex education taught in the city of Cordoba in the period 2007-2008. The production and analysis of data in this study allows theorizing the practice of teacher's formation as the setting of a transformative process of socio-sexual identities into sexgeneric-professional subjectivities, by means of the curricula contents. This process of subjectivization may either consolidate the agreed upon ways of professional legitimization, or question them in order to reinvent the profession and its sex and generic traits. The nature of this study is then comparative, and the methods used for the production of data were participant observation and content analysis of the printed material.

\section{Key words}

Sex education - Teacher training - Professional identity - Subjectivization processes - Sexualities.

\section{Introducción}

A diferencia de muchos contenidos curriculares considerados socialmente menos controversiales, la incorporación formal de la educación sexual en el sistema educativo argentino requirió de una discusión parlamentaria y la sanción de la ley 26.150 que instituyó un marco jurídico de regulación de esta labor docente. Esto da cuenta de que, pese a las modificaciones introducidas por la globalización, aún perdura el vínculo entre el estado nación moderno y la sexualidad al que refirieron Epstein y Johnson en sus análisis de las políticas sexuales en las instituciones educativas en el Reino Unido (2000). Pero a su vez indica que como resultado de disputas en el ceno de la sociedad civil, el modo en que se codifica la sexualidad en las instituciones de socialización estatal, como las escuelas, está cada vez más sujeto a transformaciones. Así, sancionada en noviembre del 2006 e instituyendo el Programa de Educación Sexual Integral (PESI) la ley formalizó e impulsó una práctica pedagógica que, por lo general, había formado parte más del currículum oculto que del manifiesto.

A partir del año 2007, tanto en Córdoba como en el resto del país, se vieron proliferar cursos de formación continua de docentes en educación sexual. Privilegiando diversos abordajes, intentaron cubrir, en primer lugar, la carencia de formación inicial - en educación sexual - de los docentes en ejercicio, y en segundo lugar, el escaso impulso y financiación que el mismo PESI le otorgó a la formación docente. Organizados por diversas agencias de formación , estos cursos requieren, no obstante, de la aprobación de sus propuestas curriculares por parte del Ministerio de Educación provincial. Este estudio, basado en el análisis comparativo de tres de dichos cursos, intenta comprender algunas 
tendencias de la cultura sexual que se les ofrece a los docentes a través de los cursos de formación en educación sexual. El foco de este artículo está puesto en la formación docente como espacio y marco de constitución y adscripción de identidades profesionales que responden a marcas genéricas y sexuales.

El artículo presenta, en primer lugar, un breve recorrido por la bibliografía local que aborda la educación sexual como un desafío relativamente nuevo para los docentes. En segundo lugar se expondrá con mayor detalle el problema informado por la discusión teórica que guió el trabajo y se señalará la metodología utilizada para generar los datos que se analizaron y en tercer lugar se expondrán los análisis y resultados.

\section{Antecedentes teóricos}

Dada la relativa novedad de la temática, investigar algún problema vinculado a la formación de docentes en educación sexual en el contexto local, implica enfrentarse a un problema disciplinar básico: la escasa bibliografía apoyada en investigación empírica para orientar el trabajo.

El currículum oculto, generalmente en el momento previo a la constitución de programas formales estatales de educación sexual, ha sido objeto de indagación por parte de algunos teóricos (Bonder y Rodriguez Giles, 1986). La práctica de la educación sexual previa a la constitución del PESI, también ha sido explorada a partir de las percepciones de los alumnos (Jones, 2009). Otros estudios que no se apoyan en diseños de investigaciones empíricas, han constituido, no obstante, un buen punto de anclaje para el activismo en la lucha por la ampliación de la ciudadanía sexual dentro del ámbito educativo (Lopes Louro, 1999).

La educación sexual como práctica pedagógica se ha vuelto en algunos casos objeto de estudio. Brevemente mencionamos aquí sólo un par de trabajos, aquellos más representativos por focalizarse sobre el mismo objeto pero siguiendo tradiciones diversas. El trabajo de Wainerman, Di Virgilio y Chami (2008) es tal vez hasta el momento, el intento de abordaje más sistemático y extensivo de la educación sexual en la Argentina. Fue realizado sobre veinticuatro escuelas de cuatro jurisdicciones diferentes con intenciones comparativas. No se realizaron observaciones de dichas prácticas sino que se entrevistaron tres profesionales de cada institución educativa (directivos, referentes y ejecutantes) con el objetivo de establecer una caracterización tipológica de las perspectivas dominantes en la materia. El trabajo se realizó siguiendo de cerca la propuesta de Michael Reiss (citado en Wainerman y cols. 2008) consistente en distinguir dos grandes posiciones filosóficas, "restrictivas" vs. "permisivas", que articulan la educación sexual. Las autoras propusieron cuatro perspectivas o tipos dominantes en los abordajes de la educación sexual (a) Educación confesional para una sexualidad con fines reproductivos, b) Educación científica para la prevención de las consecuencias de la sexualidad, c) Educación para el ejercicio de una sexualidad responsable, d) Educación para 
el ejercicio del derecho a la sexualidad). El privilegio acordado a la construcción de la tipología desdibujó la comparación entre jurisdicciones pero se analizaron y se incorporaron reflexiones sobre las estrategias de enseñanzas y los dispositivos didácticos utilizados. Tal vez la mayor limitación de este abordaje sea el descuido del carácter situado de la práctica educativa. Las identidades docentes desaparecen tras una práctica impersonal que se articula siguiendo las características principales de algunos tipos ideales. El recorte realizado por los autores deja de lado la formación (formal o informal) de los docentes en temáticas de sexualidades.

Otra línea de trabajo está constituida por los aportes de Morgade $(1992,1998)$ Alonso, Herczeg y Zurbriggen $(2008,2009)$ Ramos $(2009)$ y Greco y Entin (2009) entre otros. No se trata por cierto de una tradición homogénea ni por sus métodos, ni por sus marcos teóricos; pero tal vez convenga agruparlas por poner un énfasis mayor, tal vez principal, en el carácter situado del proceso pedagógico en la educación sexual y una comprensión política de la sexualidad. Conocidos son los aportes de Morgade (1992) en cuanto a considerar el marcador social de género como un determinante en la práctica e identidad docente en Argentina. Esta autora también ha abordado el estudio de los procesos de concientización genérica o toma de conciencia de la posición en un sistema binario de géneros en talleres con docentes (Morgade, 1998). Cabe resaltar que los primeros abordajes sobre determinantes socio-sexuales de la práctica docente en Argentina no corresponden al ámbito académico sino al ámbito del periodismo gay. En marzo de 1999, la revista NX publicaba un artículo pionero en que se abordaba en sentido amplio la discriminación en la escuela y en el que la voz de docentes gays y lesbianas aparecían como informantes principales para analizar dicha problemática (Escuela y Discriminación, [Editorial]. NX, Marzo 1999). A nivel académico el trabajo de Alonso, Herczeg y Zurbrigen (2008) basado principalmente en sus propias experiencias en el terreno de la educación sexual se inscribe en esta línea y tienen por objetivo analizar el carácter hetero-normativo de la cultura escolar pero también sus bordes y fisuras. No obstante el relato que construyen de la historia de la educación sexual en la provincia de Neuquén desde el regreso de la democracia presta escasa atención al análisis de la formación docente para centrarse en las consecuencias de los avatares políticos locales sobre la educación sexual. Aunque estas autoras prestan atención a "la producción escolar y cultural de sujetos de géneros y sexualidades" (Alonso, Herczeg y Zurbrigen, 2009, p. 213) articulando su trabajo desde una línea posestructuralista e iluminando varios problemas que afrontan en su vida laboral las y los docentes sexual y/o genéricamente disidentes, no obstante, el anudamiento y la producción de sujetos sexo-genéricos y de sujetos profesionales no es abordado en la formación docente. 
Finalmente, se puede señalar que los trabajos dedicados específicamente a abordar la formación docente, sólo muy tangencialmente tratan temas de sexualidad/es y de educación sexual. La ausencia de una política pública a nivel nacional en materia de formación de docentes en educación sexual (por lo menos hasta el año 2008) se ve reflejada en la bibliografía que aborda el tema (Alliaud y Duschatzky comp., 1998, Birgin, Dussel, Duschatzky y Tiramonti comp., 1998, Chapato y Errobidart, 2008, etc). El área disciplinar del estudio de la formación docente presenta pues un vacío en lo que respecta a la formación en educación sexual y su incidencia sobre los procesos de construcción de la identidad profesional. Sobre este terreno intenta avanzar esta comunicación.

\section{Cursos de formación docente como marcos discursivos de subjetivación sexo-genérico-profesional}

Analizar la formación, identidad y práctica docente considerando variables de orden sexuales ilumina una serie de problemáticas invisibilizadas en el campo académico local hasta fecha reciente. Lisa Smulyan (2009) nos recuerda que, en el contexto anglosajón, los escasos autores que han estudiado la experiencia docente de personas que no se identifican como heterosexuales, señalan la permanente tensión entre el orgullo por su trabajo y el temor a perderlo. La exigencia de construir una identidad profesional hetero-normada, lleva en gran cantidad de casos a los docentes Igtttb (lesbianas, gays, travestis, transgéneros, transexuales y bisexuales) a permanecer en el closet. Para algunos profesores gay, profesoras lesbianas y trans el permanente auto-control para protegerse y proteger su trabajo, reduciría la capacidad de comprometerse personalmente en las relaciones con alumnos y pares. En muchos casos generarían barreras y articularían mecanismos de distanciamiento que terminan limitando la riqueza de sus capacidades profesionales (Epstein y Johnson, 2000). Lisa Smulyan señala que:

Discursos históricamente construidos sobre la sexualidad como peligrosa (Moran, 2000), sobre los/las homosexuales como sexualizados/as (y como reclutadores o predadores) (King, 2000), y de las mujeres enseñantes como ejemplos morales - y por lo tanto asexuadas o no-sexuadas - se combinan para crear un marco conflictivo y a menudo silenciador en el cual los/as enseñantes queer negocian sus identidades. (Smulyan, 2009, p. 8)

Mi intención en este trabajo es analizar cómo los contingentes discursos de tres cursos de formación docente en educación sexual realizados en Córdoba entre el 2007 y el 2008, se erigen en marcos conceptuales hegemónicos o relativamente críticos de la hegemonía que instalan las condiciones de posibilidad a partir de las cuales se construyen y negocian identidades sexo-genérico-profesionales (Laumann y Gagnon, 1995). Enfrentando las identidades sexo-genérico-profesionales privilegiadas e incuestionables, los 
docentes no-heterosexuales deberán negociar sus identidades tal como nos lo recuerda Smulyan.

Los cursos en cuestión, fueron la posibilidad de investigar cómo en el discurso educativo de la formación de docentes se legitimaban en y para el contexto educativo sujetos discursivamente constituidos a partir de sus identidades socio-sexuales. Este proceso no podía ser observado sin prestar atención a su reverso y fue por lo tanto la ocasión de examinar cómo el mismo discurso educativo construía identidades socio-sexuales inhabitables e inhabilitadas para transitar estos espacios (Youdell, 2005, 2006). Enlazando y/o desenlazando identidades sexuales e identidades profesionales estos cursos constituyen instancias de subjetivación y sujeción en el mismo movimiento discursivo (Althusser, 2005). La importancia de focalizar en los procesos de subjetivación profesional radica en su relación con los procesos de selección escolar, reproducción social, pero también con el cambio y las articulaciones de resistencias en el ámbito educativo.

Puesto que el currículum es una selección y ordenamiento de conocimientos que establece jerarquías, analizar sus contenidos y su sistema de prioridades nos permite ver cómo la cultura escolar reproduce o modifica los significados hegemónicos. Y a partir de allí, podemos interrogar nuestro objeto de estudio para ver cómo se construyen, en estos procesos, subjetividades profesionales sexo-genéricamente caracterizadas.

No se tratará simplemente de analizar la cultura sexual vehiculizada por estos cursos y de este modo discutir el orden de reproducción escolar de significados ligados a la sexualidad, sino que intentamos captar procesos de legitimación y construcción de la identidad profesional que se acoplan a la aparente transmisión de contenidos curriculares y formulas didácticas. El desplazamiento del enfoque consiste en pasar de estudiar la reproduccióntransformación de la cultura sexual escolar en el curriculum, a estudiarla a partir de los procesos de subjetivación a los que dan lugar dichos contenidos y las prácticas pedagógicas (Althusser, 2005, Butler, 2001). Focalizamos pues sobre la regulación sexo-genérica de una actividad profesional, regulación que se presenta particularmente fuerte para el caso de las profesiones de la socialización-subjetivación, es decir para aquellos profesionales cuyo material de trabajo son otras personas. De este modo el objetivo es analizar cómo estos cursos tienen la capacidad de resolver el problema de la conversión de identidades socio-sexuales en identidades sexo-genérico-profesionales.

La selección de la muestra para el análisis siguió un criterio teórico: debían ser cursos organizados por agencias de formación docente que representen diversas posiciones dentro del mercado de la formación docente. Se tomó un caso del ámbito público, otro del ámbito privado y otro del gremio docente. También importaba que sean cursos con puntaje, es decir hayan pasado exitosamente la evaluación curricular por parte del Ministerio de la Provincia de Córdoba. Para 
la generación de datos utilizamos dos métodos según las posibilidades que ofrecieran los cursos. La observación participante con elaboración de registro de campo fue posible en los cursos presenciales (Goetz, J y LeCompte, M, 1988). En el caso del curso no presencial que analizamos, se realizó un análisis temático del contenido del material impreso.

\section{Primer curso, hetero-narrativas y efectos de silenciamiento}

De carácter no presencial, el primer curso que consideramos fue ampliamente difundido a través del periódico con mayor audiencia en la provincia, La Voz del Interior, y contaba con el aval de la Universidad Nacional de Córdoba, el Ministerio de Salud y el de Educación. Fue una de las primeras propuestas de formación docente en educación sexual posterior a la ley que no fuera organizada directamente por organismos religiosos. De los 14 fascículos publicados entre Junio y Septiembre del 2007, nos detendremos en los tres primeros pues consolidan mediante diversos mecanismos una representación fuertemente hetero-normada de sexualidad que se mantendrá en el resto de su propuesta.

Considerar las ilustraciónes del primer fascículo permite ver cómo desde la gráfica se construye para la educación sexual una continuidad simbólica con un universo cultural occidental en el que la religión, la ciencia y la disciplina institucional son referencias fundacionales. Referencias a la tentación de Adán por Eva, la masculinidad como el opuesto complementario de la feminidad, la temporalidad regulada y regular de los ciclos orgánicos, el control sobre las conductas y guiones sexuales dan inicio a un curso que privilegia una cultura escolar hetero-normativa y la presenta con una validez incuestionable (La voz del interior, 2 de Junio de 2007).

El segundo fascículo está destinado a reconocer desde el punto de vista de la anatomía, la genitalidad humana. Pero veremos cómo esta disciplina "científica" informa un discurso pedagógico que a su vez articula un sistema de sexo-género-deseo en el cual se intenta borrar cualquier fisura (Butler, J. 2001). Bajo el título "el encanto de lo distinto" puede leerse "Hombres y mujeres compartimos órganos y sistemas en los que priman las similitudes, pero las diferencias son la sal del encuentro entre los sexos. Una guía para conocernos mejor, desde el punto de vista de la anatomía" (La voz del Interior, 9 de Junio de 2007, p. 2). Una anatomía que describe dos modelos genitales y que borra cualquier cuerpo intersexuado, señala la condición corporal que valida el género, borrando cualquier identidad de género trans, y finalmente ordena una dirección del deseo, borrando cualquier identidad ligada a orientaciones homoeróticas. Si sumamos a esto una perspectiva étnica, podemos señalar que la configuración subjetiva que este curso universaliza, puede expresarse en estas dos categorías: $\mathrm{macho/varón/heterosexual/blanco} \mathrm{y} \mathrm{hembra/mujer/heterosexual/blanca} \mathrm{(Youdell,}$ D. 2005). El resto de las subjetividades prácticamente no existen. Aquellas subje- 
tividades construidas a partir de orientaciones homoeróticas serán patologizadas pues verán la indagación etiológica de su deseo, en el fascículo 12 titulado "la condición homosexual" (La voz del Interior, 18 de Agosto de 2007). Pero este discurso prácticamente no habilita voces de organizaciones o asociaciones por los derechos de minorías sexuales. Finalmente aquellas identidades ligadas a identidades de género trans, no serán mencionadas.

En el fascículo tres, la fisiología toma el relevo. El clásico estudio de Masters y Johnson es retomado para señalar las cuatro etapas del encuentro sexual (lease heterosexual) (La voz del Interior, 16 de Junio de 2007). Realizado en laboratorio, sin mayores atenciones a los guiones sexuales ni a la economíapolítica del orgasmo, este estudio fijó pautas sexuales de la cultura heterosexual, valga decir, tuvo efectos de realidad (Duncombe, y Marsden, 1996). Si bien los estudios iniciados en los años 60 por estos autores produjeron un cisma en el estudio de la sexualidad humana con perspectiva biologisista, instalando la transacción erótica en el centro del interés científico por la sexualidad y desplazando a la reproducción, no obstante, la pareja heterosexual constituyó la unidad central de análisis en el estudio, lo cual es poco coherente con la decisión teórica de considerar al placer o la función erótica como el elemento distintivo de la vida sexual (Giami, 1999). Pero, a los fines de este trabajo, es tal vez más significativo que el suplemento omita abordar los trabajos posteriores de Masters y Johnson en donde los autores, ampliando la evidencia empírica, formulan la teoría de la equivalencia general - es decir no exclusivamente genital - de las prácticas sexuales y zonas erógenas para la obtención del orgasmo. Es en este punto en donde es más evidente que el curso funciona como un marco conceptual de silenciamiento de prácticas e identidades en el cual como señalaba Smulyan los docentes Igtttb deberán negociar la legitimidad de su status profesional.

\section{Segundo curso, cotización diferencial de las identidades socio-sexuales en el mercado educativo}

Este curso no gratuito de formación de docentes en educación sexual fue diseñado por una empresa organizadora de eventos educativos. De carácter presencial, el curso convocó a varios centenares de docentes principalmente de colegios privados durante tres jornadas consecutivas en Junio del 2008. Los docentes presenciaron una serie de conferencias magistrales de distintos profesionales, psicopedagogas, sexólogos, psicólogos, etc. que ocupan el lugar de expertos en el tema y que se caracterizan por sus producciones de divulgación científica en medios radiales y prensa escrita. Si bien se podían realizar preguntas luego de cada conferencia, el mecanismo de discusión estaba estrictamente regulado por la organización del curso dada la masividad de la concurrencia.

En esta ocasión no se trata del silenciamiento, sino más bien de la deslegitimación e inhabilitación de subjetividades socio-sexuales para la práctica 
docente. Las siguientes notas de campo permiten observar un ejemplo de cómo el problema de la transformación de identidades socio-sexuales en identidades sexo-genérico-profesionales es un asunto social que se resuelve en la práctica misma de los cursos de formación docente.

Las referencias al docente homosexual aparecieron dos veces en el discurso de la psicopedagoga que ofrece la conferencia a los y las docentes. La primera vez en un análisis que lo incluía como un caso particular dentro de un proceso en el que estarían implicados todos los docentes. Para la experta, la educación sexual debe estar precedida de un proceso reflexivo de la propia sexualidad por parte del docente. Entonces el homosexual aparece como un posible, aclara, no siempre, perverso. Alguien con una sexualidad irresuelta e irreflexiva. Estas opiniones son matizadas señalando que el heterosexual también puede ser perverso.

En otra ocasión el docente homosexual es asociado al mal ejemplo o a la incapacidad de dar el ejemplo, pues señala la experta "_ tampoco podrá suplir la figura paterna en el caso de que sea necesario".

Alguien en el público formula por escrito la siguiente pregunta que es seleccionada y leída por el presentador: "_ ¿qué hacemos cuando vemos un niño homosexual?"

La conferencista responde: "_es que hay algo de lo masculino que no está pudiendo tomar". Señala que se debe avisar a los padres y trabajarlo con los especialistas. "_ no se debe dramatizar ni rotular, no se puede decir qué destino es mejor..." pero "_ el chico va a producir burlas y la va a pasar mal". En ningún momento señala la necesidad de modificar las dinámicas educativas homofóbicas, lesbofóbicas y transfóbicas para que cualquier subjetividad sexual acceda en igualdad de condiciones a constituirse en sujeto de aprendizaje. (Nota de campo)

Vemos que transformar una identidad socio-sexual en una identidad sexo-profesional es un artificio social que tiene como escenario el curso de formación docente. Algunas personas, en este caso los y las especialistas del curso, son llamados a inventar las formulas de esta transformación desde un lugar privilegiado. Aquí, las reglas de transformación, que se apoyan en teorías del psiquismo poco explicitadas, indican que no resultaría correcta la ecuación que lleva a transformar a un adulto homosexual en un docente y menos aún en un formador en educación sexual. Este marco conceptual alimenta la sospecha de supuestos abusadores que pesa sobre los docentes no-heterosexuales pero también sobre los docentes varones heterosexuales en contexto de educación temprana tal como lo señala Brailovsky (2008). Si los maestros varones heterosexuales pueden rehabilitar su dignidad profesional a partir de la demanda de 
ocupar el rol paterno (Brailovsky, 2008), como vemos, este no es el caso para los docentes no-heterosexuales en el contexto de este discurso educativo.

En este marco conceptual, el docente gay y la docente lesbiana (aunque la segunda queda invisibilizada, ni siquiera es introducida en el discurso), ocupan el polo de mayor deslegitimación sexo-profesional. Mientras tanto, el polo opuesto de subjetivación sexo-profesional docente está ocupado por la mujer-heterosexual-madre-casada, que por una alquimia que no requiere ni de un golpe de barita mágica, se transforma en docente y formadora en educación sexual. Eso explica, tal vez, el recurso retórico de la especialista, consistente en construir una identificación con su público desde la supuesta experiencia compartida de la maternidad y la conyugalidad heterosexual feliz.

Los cursos privados de formación docente son un buen escenario para indagar tendencias de la educación sexual en el contexto de la mercantilización de la educación. Los establecimientos privados, quizás más que los públicos, construyen una imagen ideal de las expectativas de los padres respecto de la educación sexual apropiada para sus hijos. El siguiente ejemplo permite ver que, en estos contextos, puede darse la pérdida del protagonismo de los intereses de los y las alumnas en provecho de los intereses de sus progenitores: "la conferencista formula la siguiente pregunta retórica: ‘qué pretenden los padres?... que no se embarace la nena!!! ...y para ello la mejor prevención es el proyecto' (se refiere a que el adolescente tenga su "proyecto de vida" alejado de la maternidad adolescente)" (nota de campo). En contextos de formación docente de corte empresarial es probable que este rasgo se repita y conforme un patrón modificando uno de los principios del PESI. La educación sexual deja de verse como un derecho que empodera a los educandos para convertirse en un producto cultural cuyo valor de consumo es negociado con padres-clientes. Aunque cabe esperar que el mercado educativo privado sea muy lábil a los cambios en las demandas de padres y alumnos, todo parece indicar que las representaciones heteronormativas constituyen aún los significados cuyo valor en el mercado educativo es menos riesgoso. Las propuestas pedagógicas feministas y queer, que intentan deconstruir tales representaciones, aparecen con un valor mercantil más dudoso y son promovidas por docentes militantes o en instituciones educativas de elite dependientes de universidades públicas.

\section{Tercer curso. Romper los consensos y reinventar la docencia, una propuesta militante}

De carácter gratuito para los afiliados y organizado por el gremio de docentes de la provincia de Córdoba, UEPC, este curso-taller diseñado a finales del 2008, respondía a estrategias pedagógica que promueven la participación. Si consideramos la apuesta pedagógica del mismo, podemos decir que, más que tratarse de un espacio de transmisión de saberes y técnicas didácticas, el 
curso intentaba constituirse en un espacio de jerarquización profesional y reforzamiento de la autonomía a partir de la reflexión y discusión con los pares.

La perspectiva de género constituyó el sesgo explícito en la construcción de un marco conceptual de habilitación de identidades sexo-profesionales. En esta propuesta el feminismo académico y militante se proponía, en primer lugar, deconstruir las identidades sexo-profesionales naturalizadas en los discursos educativos considerados hegemónicos. En segundo lugar, se intentaba recuperar el campo de posibilidades abiertas por este esfuerzo deconstructivo para el empoderamiento profesional, reinventando la profesión desde nuevos parámetros sexo-genéricos.

No obstante, hemos podido observar cómo estos intentos generan considerables disputas entre los y las docentes, por un lado, y entre las docentes y la profesora formadora de docentes, por el otro. Intentar visibilizar, desde el relativamente privilegiado lugar de la academia, cómo las identidades naturalizadas por las y los docentes participan de un sistema de dominación social sexo-genérico implica enfrentarse a los principales marcos conceptuales a partir de los cuales muchas y muchos docentes construyen sus identidades sexo-profesionales.

El intento por visibilizar las trampas que conllevaría el anudamiento de la profesión docente con otros roles sociales tradicionalmente asociados al género femenino, nos ofrece, en el caso de este curso, una clara muestra de las contradicciones a las que se enfrentan las posiciones emancipadoras. En efecto, la constelación maestra-madre-esposa fue objeto privilegiado de tales esfuerzos deconstructivos. Implicada en múltiples dinámicas de dominación - devaluación salarial por ser un salario de esposa, doble jornada laboral, devaluación de las habilidades técnico-académicas en beneficio de aquellas vinculadas al maternaje, etc. - la mencionada constelación constituye el marco conceptual hegemónico en la construcción y legitimación de la identidad profesional docente. ¿Cómo reaccionaron las docentes a las críticas al mandato materno que vehiculizaba el taller? En varias ocasiones, se produjeron fuertes reacciones en defensa de la maternidad como rol femenino, acompañada en ocasiones con repudios y llantos.

¿Debemos entender esto como una muestra de "falsa conciencia de género" entre las docentes mujeres? Tal vez conviene aceptar que, si bien innegablemente las construcciones hegemónicas de la identidad sexo-profesional constituyen la condición de posibilidad de dominaciones sexo-genéricas, no obstante, se debe reconocer que constituye también el marco a partir del cual para muchas y muchos docentes es posible habitar la profesión. Si las formas de subjetivación subyugan, debe reconocerse que también habilitan a los sujetos para la construcción de sentidos.

La tarea de deconstruir los discursos que con mayor eficacia (es decir, aquellos discursos más naturalizados) sostienen la legitimación social de la profesión docente, constituye un desafío arduo pues se enfrenta a reacciones 
y genera conflictos incluso entre los y las docentes. Apostar por cuestionar el consenso social establecido en la construcción de sujetos profesionales sexogenéricamente atravesados, constituye el rasgo propio de una formación docente militante que busca fortalecer la autonomía profesional de-construyendo las bases discursivas de la dominación pero que se enfrenta necesariamente a reacciones conservadoras.

\section{Conclusiones}

Cuando el estado intenta monopolizar la socialización sexual a través del Programa de Educación Sexual Integral, distintos sectores corporativos (iglesia, profesionales de la salud, movimientos sociales, etc.) se disputan la posibilidad de influir sobre esta práctica manipulando la constitución de la identidad de los agentes del sistema educativo. Focalizar sobre los anudamientos sexogenéricos en el proceso discursivo de subjetivación profesional docente, permite identificar una activación de la tensión entre tendencias reproductivistas y transformativas del sistema sexo-género-deseo a partir de la ley 26.150. Podemos señalar pues, como conclusión general, que habría dos modelos opuestos para resolver, en los cursos de formación docente, el problema de la conversión de identidades socio-sexuales en identidades sexo-genérico-profesionales.

El primero de estos modelos incluye aquellos intentos de resolver mediante una ficción esencialista esta exigencia social. Los marcos conceptuales de subjetivación que naturalizan identidades sexo-genérico-profesionales silencian o deslegitiman aquellas identidades socio-sexuales que son vistos como restos no profesionalizables (gays, lesbianas, travestis, transexuales, transgéneros, bisexuales, intersexuales). En la actualidad este polo articula la cultura profesional con las identidades heterosexuales como es el caso en el primer y el segundo curso de formación docente analizados. El mayor desplazamiento en la actualidad se da a nivel del marcador de género, legitimando y jerarquizando en muchos casos a los profesionales varones.

En el polo opuesto pueden ubicarse los esfuerzos por resolver políticamente el mismo problema. Políticamente, es decir, visibilizando que los conflictivos y silenciadores marcos conceptuales en los cuales los profesores, tanto noheterosexuales como heterosexuales, deben negociar sus identidades profesionales, son históricos y contingentes, no matemáticos e inmutables. Este modelo cuestiona la hegemonía discursiva de-construyendo el artificio mismo de la subjetivación sexo-genérico-profesional. En el tercer curso, analizado, reinventando la profesión docente desde un discurso de empoderamiento feminista proclive a entablar alianzas con reivindicaciones del movimiento LGTTTB.

Proponemos aquí la conveniencia de clasificarlos en modelos de subjetivación sexo-genérico-profesional naturalistas vs. genealógicos o deconstructivos.

Para concluir, vale considerar que fenómenos como el ausentismo, el abandono, la migración escolar y el fracaso escolar, la alienación y rutinización 
profesional y el burn-out docente, pero también la violencia y discriminación sexual escolar, deberían ser estudiados a la luz de procesos complejos de subjetivación en los que se entrelazan marcadores de géneros, sexualidades y roles profesionales.

\section{Referencias Bibliográficas}

- Alliaud y Duchatzky (comps.) (1998) Maestros: Formación, práctica y transformación escolar, Ed. Miño y Dávila, Buenos Aires.

- $\quad$ Alonso, G. Herczeg, G y Zurbriggen, R. (2009) Cuerpos y sexualidades en la escuela. Interpelaciones desde la disidencia, en Alejandro Villa (comp.) Sexualidad, relaciones de género y de generación, Ed. Noveduc, Buenos Aires, pp. 213-239.

- Alonso, G. Herczeg, G y Zurbriggen, R. (2008) Talleres de educación sexual. Efectos del discurso heteronormativo, en Morgade, $G$ y Alonso, $G$ (comps.) Cuerpos y sexualidades en la escuela, Ed. Paidós, Buenos Aires, pp. 251-272.

- $\quad$ Althusser, L. (2003) Ideología y Aparatos Ideológicos de Estado, en Slavoj Žižek (comp.) Ideología. Un mapa de la cuestión, Ed. Fondo de Cultura Económica, Buenos Aires, pp. 115-155.

- Birgin, Dussel, Duschatzky y Tiramonti (comps.) (1998) La formación docente, Ed. Troquel, Buenos Aires.

- Bonder, G y Rodriguez, E. (1986) Estereotipos sexuales en la educación primaria. CEM, Buenos Aires.

- Brailovsky, D. (2008) Educación infantil y masculinidades. El caso de los maestros jardineros varones, en Morgade, G y Alonso, G (comps.) Cuerpos y sexualidades en la escuela. De la normalidad a la disidencia, Ed. Paidós, Buenos Aires, pp.113-147.

- Butler, J. (2001) El género en disputa. El feminismo y la subversión de la identidad, Ed. Paidos, México.

- Chapato y Errobidart (2008) Hacerse docente. Las construcciones identitarias de los Profesores en sus inserciones laborales, Ed. Miño y Dávila, Buenos Aires.

- Duncombe, J y Marsden, D. (1996) Whose orgasm is this anyway? "Sex work" in LongTerm heterosexual couple relationships, en Weeks, J y Holland, J, (comps.) Seal cultures, communities and values, Ed. Macmillan, Oxford, pp.220-238.

- Entin, C. y Greco, M. B. (2008) La información no alcanza para el cuidado. Una experiencia de Teatro-foro en la formación docente, en Morgade, G y Alonso, G (comps.) Cuerpos y sexualidades en la escuela, Ed. Paidós, Buenos Aires, pp. 273-289.

- Epstein, D y Johnson, R. (2000) Sexualidades e institución escolar, Ed. Morata, Madrid.

- Escuela y Discriminación [Editorial]. (1999, Marzo) revista NX, N·64, pp.20-23, Ed. Grupo Nexo Asociación Civil, Buenos Aires.

- Giani, A. (1999) Cent ans d'heterosexualité, en Actes de la recherché en sciences sociales, V. 128, Paris, pp.38-45.

- Goetz, J. y Lecompte, M. (1988) Etnografía y diseño cualitativo en investigación educativa, Ed. Morata, Madrid.

- Jones, D. (2009) ¿De qué hablamos cuando hablamos de sexualidad? Educación sexual en escuelas de nivel secundario antes de la Ley de Educación Sexual Integral de la Argentina, en Argumentos, revista de crítica social, N·11, Instituto Gino Germani. (www. revistaargumentos.com.ar consulta: Octubre 2009). 
- Laumann, E. y Gagnon, J. (1995) A Sociological Perspective on Sexual Action, en Conceiving Sexuality. Approaches to Sex Research in a Postmodern World, Routledge, New York, pp. 183-213.

- La voz del Interior: Educación sexual: el desafío de la hora. Miradas sobre la sexualidad [Editorial]. (2007, 2 de Junio) La Voz del Interior pp.2-3.

- $\quad$ La sexualidad, de ayer a hoy [Editorial]. (2007, 2 de Junio) La Voz del Interior pp.4-5.

- $\quad$ El encanto de lo distinto [Editorial]. (2007, 9 de Junio) La Voz del Interior p. 2.

- Los órganos genitales [Editorial]. (2007, 9 de Junio) La Voz del Interior pp.4-5.

- Todo el cuerpo para una respuesta [Editorial]. (2007 16 de Junio) La Voz del Interior p. 2.

- $\quad$ Fases de la respuesta sexual [Editorial]. (2007, 16 de Junio) La Voz del Interior pp.4-5.

- Del estigma a la aceptación [Editorial]. (2007, 18 de Agosto) La Voz del Interior p. 3.

- Definiciones y características [Editorial]. (2007, 18 de Agosto) La Voz del Interior pp.4-5.

- Lopes Louro, G. (1999) Pedagogias da sexualidade, en O corpo educado, Ed. Autêntica, Belo Horizonte, pp-9-34.

- Morgade, G. (1998) La formación docente en la perspectiva de género: un proceso especular, en La Formación docente. Cultura, escuela y política, debates y experiencias, Ed. Troquel, Buenos Aires, pp.121-127.

- Morgade, G. (1992) El determinante de género en el trabajo docente de la escuela primaria, Ed. Miño y Davila, Buenos Aires.

- Ramos, G. (2009) Discurso/s que construyen sexualidades en escuelas medias de la Ciudad de Buenos Aires, en Alejandro Villa (comp.) Sexualidad, relaciones de género y de generación, Ed. Noveduc, Buenos Aires, pp. 139-169.

- Smulyan, L. (2006) Constructing teaching identities, en The sage Handbook of Gender and Education, Sage publications, (Consulta: Septiembre 2009).

- Youdell, D. (2006) Subjectivation and performative politics - Butler thinking Althusser and Foucault: intelligibility, agency and the raced-nationed-religioned subjects of education, en British Journal of Sociology of Education, V.27, N-4, Routledge, pp. 511-528.

- Youdell, D. (2005) Sex-gender-sexuality: how sex, gender and sexuality constellations are constituted in secondary schools, en British Journal of Sociology of Education. Vol 17, N·3, Routledge, pp. 249-270.

- Wainerman, C, Di Virgilio, M. y Chami, N. (2008) La escuela y la educación sexual, Ed. Manantial, Buenos Aires. 